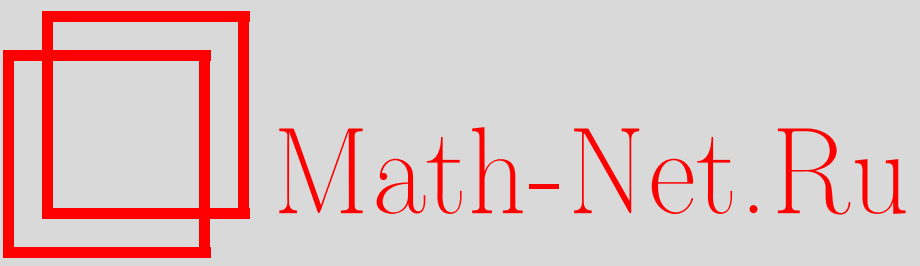

А. Я. Белов, О рациональности рядов Гильберта относительно свободных алгебр, УМН, 1997, том 52, выпуск 2, 153-154

DOI: https://doi.org/10.4213/rm827

Использование Общероссийского математического портала Math-Net.Ru подразумевает, что вы прочитали и согласны с пользовательским соглашением

http://www.mathnet.ru/rus/agreement

Параметры загрузки:

IP: 3.85 .5 .30

26 апреля 2023 г., $11: 30: 22$ 


\title{
О РАЦИОНАЛЬНОСТИ РЯДОВ ГИЛЬБЕРТА ОТНОСИТЕЛЬНО СВОБОДНЫХ АЛГЕБР
}

\author{
А.Я. БЕЛОВ
}

Введение. Основной резултат данной работы заключается в следующем утверждении.

Теорема. Пусть $A$ - конечно-порожденная относительно свободная PI-алгебра над бесконечным полем. Тогда $H_{A}-$ яяд Гильберта алгебры А-рационален.

При доказательстве рациональности рядов Гильберта естественно воспользоваться известным резултатом А.Р.Кемера [1] о представимости относителшно свободных алгебр над бесконечным полем. Однако существуют примеры представимых алгебр с трансцендентным рядом Гильберта. Необходимо использоание специфики относительно свободного случая.

Доказательство основного результата. Пусть $A$ - относительно свободная алгебра, она представима так, что $A \in \operatorname{Var}\left(A^{\prime}\right)$, где $A^{\prime}$ - конечномерная алгебра. Обозначим через $\left\{A^{(i)}\right\}$ набор алгебр с меньшей полупростой частью, чем алгебра $A^{\prime}$. Точнее, $A^{(i)}=E_{i} A E_{i}$, где $E_{i}-$ пирсовская компонента единицы. Пусть $A_{0}$ - прямая сумма простых компонент $A_{j}$ алгебры $A^{\prime}$, которые мы иногда будем назьвать клетками. Итак, $A_{0}=\oplus_{j=1}^{s} A_{j} ; A=A_{0}+R, R=R(A)$ - радикал алгебры $A$. Пусть $F\langle\theta\rangle$ - относителшно свободная алгебра, порожденная элементами $\theta_{i}$. Через $A(d)$ обозначается алгебра, получаемая из $A_{0}$ свободным присоединением радикала степени $d$ в многообразии $\operatorname{Var}(A)$. Иными словами, $A(d)=A_{0} * F\langle\theta\rangle /\left(\theta^{(d)}+T(A)\right)$, где $\theta^{(d)}$ обозначает степень идеала, порожденного $\left\{\theta_{i}\right\}$.

Предложение 1. а) Пусть $f \notin T\left(A^{(i)}\right)$ для некорого $i$. Тогда имеются следствие $g \in T(f)$ и әлементы $b_{i}$, a также $a_{j} \in A_{j}, j=1, \ldots, s$ ( $j$ пробегает полный набор значений) такие, что $g\left(a_{1}, \ldots, a_{s}, b_{1}, \ldots, b_{q}\right) \not \equiv 0 \bmod (T(A))$.

б) Если $E_{l m}^{j}$ означает матричную единицу, отвечающую $j$-й клетке - алгебре $A_{j}$, то в качестве $a_{j}$ можсно взять произведения вида

$$
Q_{j}\left(E_{11}^{j} E_{11}^{j} E_{11}^{j} E_{12}^{j} E_{21}^{j} \cdots E_{1 m}^{j} E_{m n}^{j} E_{n 1}^{j} \ldots\right)^{t} T_{j}
$$

(между прокладками $E_{1 m}^{j}$ и $E_{n 1}^{j}$ встречаются всевозможные матричные единиubl $\left.E_{m n}^{j}\right)$.

ЗАмечАниЕ. Если $E_{m n}^{j}$ заменить на другое $E_{m^{\prime} n^{\prime}}^{j^{\prime}}$, то произведение станет нулевым. Поэтому если выбрать набор из разных сомножителей $E_{m n}^{j}$ в произведении $g$ и проальтернировать, то результат альтернирования будет равен исходному многочлену $g$.

Основная идея доказательства рациональности ряда Гильберта состоит в нахождении $T$-идеала $I$ такого, что $H_{I}$ рационален. Тогда $H_{A}=H_{I}+H_{A / I}$, и мы приходим к доказательству рациональности $H_{A / I}$. Остается воспользоваться свойством обрьва возрастающих цепей для $T$-идеалов. Кроме того, заметим, что нам достаточно также найти конечный набор $T$-идеалов с нулевым пересечением. В самом деле: если $I \cap J=0$, то $H_{A / I}+H_{A / J}=H_{A}$. В случае нескольких идеалов проходит очевидная индукция.

Итак, мы строим либо $T$-идеал с рациональным рядом Гильберта, либо конечньй набор $T$-идеалов с нулевым пересечением.

Основные обозначения. Мы следуем работе А.Р. Кемера. Пусть b1 $(A)$ обозначает $\operatorname{dim}\left(A_{0}\right), \quad c(A)$ - индекс нильпотентности $R\left(A^{\prime}\right), \quad S_{\Lambda}(f)$ - результат альтернирования полилинейного тождества $f$ по набору переменных $\Lambda, b(A)$ - максималшное целое $b$ такое, что для любого числа $k$ наборов $\Lambda_{i}$ по $b$ переменных в каждом существует $g$ такое, что $S_{\Lambda_{1}} \ldots S_{\Lambda_{k}}(g) \not \equiv 0$ $\bmod (T(A))$. Для $T$-идеала $\Gamma$ определяется аналогично $b(A, \Gamma)$, только на $g$ накладывается дополнительное условие принадлежности $g \in \Gamma$. Ясно, что $b(A)<\mathrm{b} 1(A)$. Положим $d(A)=q+1$, 
$q$ - минимальное целое такое, что существуют многочлен $g$ и $q$ наборов из $b(A)+1$ переменной в каждом такие, что $S_{\Lambda_{1}} \cdots S_{\Lambda_{q}}(g) \not \equiv 0 \bmod (T(A))$. Аналогично определяется относительная величина $d(A, \Gamma)$.

Из замечания после предложения 1 вытекает

ПреДЛОЖЕНИЕ 2. Если $b(A)<\mathrm{b} 1(A), \operatorname{mo} T(A)=\cap T\left(A^{(i)}\right)$.

Итак, мы можем считать, что $b(A)=\mathrm{b} 1(A)$. В этом случае нетрудно убедиться, что $d(A)<c(A)$. Пусть $f \in \bigcap_{i} T\left(A^{(i)}\right)$ и $f \notin T(A)$. Тогда $b(A)=b(A, f)$ и $d(A, f)<d(A)<c(A)$. Пусть $d$ - максимум таких $d(A, f)$, и пусть $d<c(A)$. В этом случае тем же методом, что и в работе А. Р. Кемера, доказьвается следующее редукционное

ПреДЛОЖЕНИЕ 3. Пусть $b(A)=\mathrm{b} 1(A)$, и пусть $d<c(A)$. Тогда $T(A(d))$ u $T\left(A^{(i)}\right)$ образуют конечный набор $T$-идеалов с нулевым пересечением.

Редукционные леммы позволяют считать, что $\mathrm{b} 1(A)=b(A)=b, d(A)=c(A)=c$. Пусть множество $\Lambda_{0}$ состоит из $b$ переменных, множества $\Lambda_{i}$, где $i=1, \ldots, c-1$, состоят из $b+1$ переменной, $I-T$-идеал, порожденнный многочленами, кососимметричными по каждому набору переменных из группы $\Lambda_{j}$. В силу сказанного выше, $I \neq 0$. Покажем, что этот $T$-идеал искомшій, т.е. обладает структурой нётерова модуля.

Прежде всего отметим, что в каждом $\Lambda_{i}$, где $i=1, \ldots, c-1$, должно находиться по одной радикальной компоненте алгебры $A^{\prime}$, поэтому в $\Lambda_{0}$ могут подставляться только полупростые части элементов. Остаеться воспользоваться следующими предложениями:

ПредЛОЖениЕ 4. Пусть $A-$ - редставимая ассоџиативная алгебра, $\widehat{A}-$ расширение А следами. Тогда $\widehat{A}$ конечномерна над иентром.

ПреДЛОЖенИЕ 5 (Ю.П. Размыслов). Пусть $A-$ алгебра $(n \times n)$-матрии, $f(\vec{x}, \vec{y})-$ многочлен, кососимметричный по $x_{i}, i=1, \ldots, n^{2}$. Тогда $\sum_{i} f\left(x_{1}, \ldots, a x_{i}, \ldots, x_{n^{2}}, \vec{y}\right)=$ $n \cdot \operatorname{Tr}(A) \cdot f(\vec{x}, \vec{y})$.

Из предложения 5 легко вьводиться следующее

ПредЛОЖЕнИЕ 6. Пусть $f$ кососимметричен по наборам переменных $\Lambda_{i}$, описаннных выше, $\left\{x_{m}\right\}$ - набор переменных из $\Lambda_{0}, m=1, \ldots, b$. Тогда $\operatorname{Tr}(A) \cdot f(\vec{x}, \vec{y})=$ $\sum_{i} f\left(x_{1}, \ldots, a x_{i}, \ldots, x_{b}, \vec{y}\right)$.

Здесь след понимается с кратностями, элемент, отвечающий клетке, умножается на ее размер.

Таким образом, для случая характеристики нуль рациональность рядов Гилберта доказана. В случае бесконечного поля положителњной характеристики проходят почти те же рассуждения (представимость конечно порожденных относителшно свободных алгебр над бесконечным полем доказана А.Р. Кемером в 1990 г.). Единственная дополнительная трудность связана с тем, что размер клетки может делиться на $p$-характеристику основного поля. В этом случае вместо формулы $(*)$ следует воспользоваться формулой

$$
\sum_{i} f\left(x_{1}, \ldots, A x_{i} B, \ldots, x_{n^{2}}, y\right)=\operatorname{Tr}(A \otimes B) \cdot f(x, y),
$$

в которую множитель $n$ не входит. При этом итоговые “глобалњные" следы будут учитываться без кратностей.

ЗАмечАниЕ. Фактически мы вывели рациональность рядов Гильберта из представимости.

$$
\text { СПИСОК ЛИТЕРАТУРЫ }
$$

[1] Кемер А.Р. // Алгебра и логика 1988. Т. 27. № 2. С. 274-294. 